\title{
Character of Microbial Engraftment Following the Fecal Microbiota Transplantation in Patients with Inflammatory Bowel Diseases
}

Evgenii I. Olekhnovich ( $\sim$ jeniaole01@gmail.com )

Federal Medical and Biological Agency of Russia

Tatyana N. Kalachnyuk

Federal Medical and Biological Agency of Russia

Dmitry E. Fedorov

Federal Medical and Biological Agency of Russia

Elena S. Zhgun

Federal Medical and Biological Agency of Russia

Ksenia M. Klimina

Federal Medical and Biological Agency of Russia

Vladimir A. Veselovsky

Federal Medical and Biological Agency of Russia

Daria I. Boldyreva

Federal Medical and Biological Agency of Russia

Roman I. Matorin

Federal Medical and Biological Agency of Russia

Svetlana V. Lyamina

Federal Medical and Biological Agency of Russia

Dmitriy N. Andreev

A.I. Evdokimov Moscow State University of Medicine and Dentistry, Ministry of Healthcare of the Russian Federation

Igor V. Maev

A.I. Evdokimov Moscow State University of Medicine and Dentistry, Ministry of Healthcare of the Russian Federation

Alexander V. Pavlenko

Federal Medical and Biological Agency of Russia

Vadim M. Govorun

Federal Medical and Biological Agency of Russia

Elena N. Ilina

Federal Medical and Biological Agency of Russia 


\section{Research Article}

Keywords: Microbial Engraftment, Fecal Microbiota Transplantation, Inflammatory Bowel Diseases

Posted Date: October 27th, 2021

DOl: https://doi.org/10.21203/rs.3.rs-999905/v1

License: (c) (i) This work is licensed under a Creative Commons Attribution 4.0 International License. Read Full License 


\title{
Character of microbial engraftment following the fecal microbiota transplantation in patients with inflammatory bowel diseases
}

\author{
Evgenii I. Olekhnovich ${ }^{1, *+}$, Tatyana N. Kalachnyuk ${ }^{1,+}$, Dmitry E. Fedorov ${ }^{1}$, Elena S. \\ Zhgun $^{1}$, Ksenia M. Klimina ${ }^{1}$, Vladimir A. Veselovsky ${ }^{1}$, Daria I. Boldyreva ${ }^{1}$, Roman I. \\ Matorin ${ }^{1}$, Svetlana V. Lyamina ${ }^{1,2}$, Dmitriy N. Andreev ${ }^{2}$, Igor V. Maev ${ }^{2}$, Alexander V. \\ Pavlenko ${ }^{1}$, Vadim M. Govorun ${ }^{1}$, and Elena N. Ilina ${ }^{1}$ \\ ${ }^{1}$ Federal Research and Clinical Centre of Physical and Chemical Medicine, Federal Medical and Biological Agency \\ of Russia, Moscow, Russian Federation \\ ${ }^{2}$ A.I. Evdokimov Moscow State University of Medicine and Dentistry, Ministry of Healthcare of the Russian \\ Federation, Moscow, Russian Federation \\ *jeniaole01@gmail.com \\ +these authors contributed equally to this work
}

\begin{abstract}
Fecal microbiota transplantation (FMT) targeting gut microbiota has recently been applied in treatment of Crohn's disease (CD) and ulcerative colitis (UC). However, only a fraction of patients respond positively to this therapy. In this regard, it is gaining popularity to study the combinations of approved medications and FMT to increase the effectiveness of therapy. Here we present a pilot longitude project for the study of FMT application combined with the anti-inflammatory therapy in CD ( $\mathrm{n}$ $=8)$ and UC $(n=6)$ patients. Using the shotgun sequencing of stool samples $(n=56)$ we evaluated the temporal changes in the taxonomic and functional structure of the patients' metagenomes and donor-derived microbes engraftment due to the FMT procedure. According to our analysis, the gut microbiota was changed both in CD and UC patients whereas reshaping nature in experimental groups was differ. Only in the UC group the shift of gut microbiota composition towards donor and the donor-derived microbes engraftment were more extensive in comparison with the CD group. Moreover, we observed that beneficial and prevailing in human population microbes such as Faecalibacterium prausnitzii, Bifidobacterium adolescentis, Eubacterium rectale, and others are the best colonizers. The difference in the microbial engraftment can be explained by differences in the pathogenesis of CD and UC. On the other hand, mucosal and intestinal lumen conditions in the UC group may have been better corrected due to the proposed treatment which allowed the donor microbes to colonize these patients more successfully. In addition, the evolutionary formed ecological relationship between the gut microbiota and the host can be linked with the FMT therapeutic effect.
\end{abstract}

\section{Introduction}

Inflammatory bowel diseases (IBD) including Crohn's disease (CD) and ulcerative colitis (UC) are chronic diseases of the gastrointestinal tract and affect about $15 \%$ of the world's human population ${ }^{1}$. The pathophysiology of IBD is complex, and the etiology remains unclear. In UC, inflammation is spread throughout the colon mucosa ${ }^{2}$, while in CD, inflammation can affect any part of the gastrointestinal tract, but primarily the terminal ileum and colon ${ }^{3}$. Current concepts confess the critical role of gut microbiota ${ }^{4}$. Moreover, microbial-based approaches have come to be viewed by the clinical community as promising novel therapy strategies of IBD.

Currently fecal microbiota transplantation (FMT) has been approved as an alternative treatment Clostridioides difficile infection (CDI). The efficacy of this method approaches $90 \%$ while maintaining a relatively high safety degree ${ }^{5}$. At the same time, there is increasing evidence of a FMT therapeutic effect in IBD ${ }^{6,7}$. However, despite the promising nature of the method, FMT shows reduced efficiency in IBD in comparison with CDI. In this regard, it is gaining popularity to study of approved therapy schemes and FMT combinations in order to increase the number of positive treatment outcomes ${ }^{8-11}$.

Here we present our experience with the use of FMT in combination with anti-inflammatory medications in the IBD patients treatment. We assess the changes of the patient's gut microbiota structure towards the donor and the healthy population in the process of decreased disease activity. We also submit data on the identified differences in the nature of the patient's gut microbiota changes and donor-derived microbes engraftment in our experimental cohort, depending on the diagnosis. 


\section{Results}

\section{Treatment strategy and assessment of patient's clinical conditions}

CD and UC patients $(n=14)$ admitted to the gastroenterology department of Federal Research and Clinical center of PhysicalChemical Medicine were included in the study. Based on the patient's clinical surveys, the disease activity index was calculated: HBI for CD patients, SCCAI for UC patients. For $7 \mathrm{CD}$ patients, the disease activity index was HBI $=5.4 \pm 0.8$ while the disease activity index was SCCAI $=6.7 \pm 0.8$ in the UC group. The CD5 patient's condition was characterized by worse general health, severe abdominal pain, loose stools with a frequency of $>20$ times a day (HBI $>30$ ).

In addition, laboratory parameters for C-reactive protein $(\mathrm{CRP} ; \mathrm{CD}=4.5 \pm 3.6 \mathrm{mg} / \mathrm{L} ; \mathrm{UC}=6.0 \pm 4.3 \mathrm{mg} / \mathrm{L})$ and calprotectin $(\mathrm{CD}=70.4 \pm 29.9 \mu \mathrm{g} / \mathrm{L} ; \mathrm{UC}=246.3 \pm 119.9 \mu \mathrm{g} / \mathrm{L})$ were assessed on admission to the hospital. It should be noted that the condition of 4 patients was complicated by extraintestinal manifestations such as arthritis ( $3 \mathrm{CD}$ and $1 \mathrm{UC}$ ). $2 \mathrm{CD}$ patients had an intestinal surgery history. Based on the assessment of the clinical condition and actual clinical guidelines, a basic therapy was prescribed (or corrected) for each patient, which included 5-ASA monotherapy (for 4 CD and 5 UC patients) or 5-ASA + glucocorticoids combination (for $4 \mathrm{CD}$ and $1 \mathrm{UC}$ patients).

The donor fecal solution was infused into the jejunum using an endoscope according to described protocol (see Materials and methods section). Non-pooled biological material obtained from 4 donors was used to treat both CD and UC patients. However, fecal samples from 2 additional donors were used in the CD group only. According to medical indications, the patients basic therapy after the FMT procedure has been adjusted: for UC3, glucocorticoids were added on day 5 after FMT; for UC33, glucocorticoids were removed after FMT. The assessment of the patient's overall clinical conditions, as well as the disease activity indices calculation was performed on the 30th day after the FMT procedure.

Reduced disease activity for all patients included in the study has been detected: HBI $3.0 \pm 3.0$ (Wilcoxon rank sum test, $\mathrm{p}<0.001$ ), SCCAI $1.7 \pm 1.2$ (Wilcoxon rank sum test, $\mathrm{p}<0.05$ ). For CD5 patients, HBI was a decrease from 38 to 10. After the treatment, the values of CRP patient levels for CD and UC were $2.7 \pm 2.0$ and $5.5 \pm 4.66 \mathrm{mg} / \mathrm{L}$, respectively, as well as calprotectin levels were $37.8 \pm 15.2$ and $79.6 \pm 53.46 \mu \mathrm{g} / \mathrm{L}$, respectively. There was a tendency for a decrease in these laboratory parameters, however, only calprotectin in CD patients showed a statistical significant decrease (Wilcoxon signed-rank test, $\mathrm{p}<0.01)$ in comparison to pre-treatment state. Thus, the proposed treatment allowed steroid-free clinical remission for $4 \mathrm{CD}$ patients and $5 \mathrm{UC}$ patients on the 30th day of observation while steroid-induced remission was achieved only for 2 CD patients. It's worth noting that only UC22 demonstrated increased disease activity after achieving clinical remission during the further observations.

The summary clinical information about IBD patients included in the study was presented in Table 1 . The study design scheme is shown in Figure 1.

\section{General changes in gut microbiota composition of patients due to FMT over time}

Stool samples were received from 14 patients as well as from 6 donors. The patient's samples were collected in the time series format: before and within 1 month after FMT. However, not all patient stool samples were collected/sequenced at all 5 time points due to organizational or technical problems. In total, 56 stool samples were collected: 24 for CD, 24 for UC, and 8 for all donors. The experimental design scheme is presented in Figure 1A. The stool samples collecting scheme for each patient is presented in Figure 1B.

Sequencing of the samples yielded $8.0 \pm 5.7 \mathrm{mln}$ of $125 \mathrm{bp}$ paired preprocessed reads per sample. The summary data about obtained stool metagenomes and sequencing statistics is presented in Supplementary Table S2. The MetaPhlAn3 method based on unique clade-specific markers allowed us to identify 423 species belonging to 10 phylums in all patient metagenomes. The MetaPhlAn3 species-level taxonomic profiles of metagenomes is presented in Supplementary Table S3. The top observed phylums included Firmicutes, Bacteroidetes, Actinobacteria, Proteobacteria, Verrucomicrobia, and unclassified Viruses. The distribution of top observed phulums relative abundances across obtained metagenomes were presented in Figure 2A.

The NMDS bi-dimensional plot obtained by using Aitchison distance and species-levels taxonomic profiles of metagenomes is presented in Figure 2B. It shows the clasterization of the stool taxonomic profiles by disease. Samples from the healthy donors shifted to the left while CD patients samples to right. The UC samples have an intermediate position between healthy donors and $\mathrm{CD}$. Analysis of the variance using PERMANOVA revealed that the disease (CD, UC, or healthy donors) was significantly linked to the taxonomic composition $\left(\mathrm{R}^{2}=0.09\right.$, adj. $\mathrm{p}<0.001$, Aitchison distance, 10000 permutations).

Changes in microbial richness (alpha-diversity) of the patient stool metagenomes after FMT was not detected (see Figure 2C). Shannon index fluctuates within the healthy donors values range. In addition, the alpha-diversity value did not significantly differ in healthy donors and pre-FMT patients $(2.5 \pm 0.4$ in the healthy donors vs $2.4 \pm 0.5$ in the CD group and $2.5 \pm 0.5$ in the UC group). However, a change in the microbial content after FMT was detected (Figure 2D; Spearman correlation $\mathrm{p}<$ 0.001 in both cases). Greater alterations are detected between the same patient samples than can be observed between the same donor samples. 


\section{General changes in gut microbiota composition of patients due to FMT over time in context of IBD patients and healthy persons from human population}

To identify the direction of microbial content changes, we analyzed the movement of a patient's gut microbial compositions over time in the context of 220 external metagenomes collected from healthy persons and IBD patients ${ }^{12}$. The taxonomic profiles of additional metagenomes are presented in Supplementary Table S4. According to the analysis (see Figure 3A), the healthy persons and donors are concentrated in the left side of the $\mathrm{x}$-axis (describes more than $50 \%$ of the total variance) while the IBD patients' taxonomic profiles shifted to the right. It is worth noting that $\mathrm{CD}$ patients are shifted to the right more pronounced in comparison with UC patients (similar to Figure 2B).

In addition, the directions of increasing abundance in respective microbial species were identified (see Figure 3B). Movement to the right size of $\mathrm{x}$-axis is associated with abnormal composition characterized by the increasing pro-inflammatory and oral species including unknown Roseburia sp CAG 471, Enorma massiliensis, Rothia mucilaginosa et al. At the same time, increasing of anti-inflammatory species and species commonly presented in human population such as Faecalibacterium prausnitzii, Ruminococcus bromii, Bifidobacterium adolescentis and others associated with movement to the left-side of x-axis.

Thus, we will associate the aggravation of the abnormal gut microbiota composition with movement to the right along the Figure 3A x-axis, while movement to the left will mean normalization (see Figure 3C). According to obtained results, $1 \mathrm{CD}$ patient (13\%) and 4 UC patients (67\%) showed a leftward shift in the X-axis over time. It is worth noting, only the UC group demonstrated statistical significant movement (see Figure 3D, Spearman correlation $\mathrm{p}<0.01$ ). At the same time, normalization of a gut microbial composition of IBD patients is associated with movement towards the donor (see Figure 3E). $2 \mathrm{CD}(25 \%)$ patients and 5 UC (83\%) patients have been shown shift towards donor whereas statistically significant movement was detected only for the UC group (see Figure 3F; Spearman correlation $\mathrm{p}<0.01$ for UC group).

It is worth noting that we were able to associate gut microbial content normalization with a decrease in disease activity only in the UC group while CD patients, in general, showed a significant clinical improvement in the absence of movement to the healthy-like composition.

\section{Discovery of microbial biomarkers associated with changes in gut microbiota composition of patients due to FMT and anti-inflammatory therapy}

The Songbird approach was used for discovery of microbial biomarkers associated with changes in gut microbiota composition of patients due to FMT and anti-inflammatory therapy. The results showed a log-fold change in features with respect to sampling points. Further, the bacterial taxa are listed in decreasing order of log-fold changes.

According to the obtained results, the pro-inflammatory and oral microbial species including Veillonella parvula, Clostridium innocuum, Veillonella atypica, Haemophilus parainfluenzae et other had a negative association with observed time in the CD group while the butyrate-producing and generally healthy-associated microbes such as B. adolescentis, P. copri, Alistipes finegoldii et other (excluding $R$. gnavus) are associated positively (see Figure 4A). Thus, we did not detect a continuous movement of patient microbial profiles over time in the context of the log-ratios of present biomarkers (see Figure 4B; Spearman correlation, $\mathrm{p}>0.05$ ). In the 2 nd time point, the patient's microbial profiles show a shift to the positively associated biomarkers (Wilcoxon signed-rank test, $\mathrm{p}<0.01$ ), however in the $3 \mathrm{rd}$ and 4 th time points returned to the pre-FMT state. It is clear that CD patients show a short-term normalization of the microbiota. However, it is possible that the gut microenvironment of these patients does not allow these positive changes to be long-term.

Also, the similar shift patterns of the UC patient taxonomic profiles were detected. The abnormal microbial content was negatively associated with time points while the healthy-related content - positively (see Figure 4C). However, movement in the log-ratios context of observed biomarkers shows clearly differences across the observation time (see Figure 4D; Spearman correlation, $\mathrm{p}<0.001)$. Thus, UC patients show temporal shifts between two microbial states.

The observed taxonomic changes were the cause of functional shifts in the UC group but not in the CD group. According to our analysis, in the UC group, the 7 KEGG pathways were overrepresented after FMT in comparison with the pre-FMT state. This list included ko01100: Metabolic pathways (adj. p < 0.01), ko01110: Biosynthesis of secondary metabolites (adj. p < 0.01), ko01130: Biosynthesis of antibiotics (adj. p < 0.01), ko01230: Biosynthesis of amino acids (adj. $\mathrm{p}<0.01$ ), ko00500: Starch and sucrose metabolism (adj. p < 0.01), ko02040: Flagellar assembly (adj. p < 0.01), ko00562: Inositol phosphate metabolism (adj. $\mathrm{p}<0.01$ ). KEGG orthology groups distributions across obtained metagenomes are presented in Supplementary Table S5.

\section{Detection of donor-derived microbes in the post-FMT metagenomes of IBD patients}

To study the contribution of the donor-derived bacteria to the changes described above in the taxonomic profiles of the patient's intestinal microbiota, the RECAST approach was used. This method is based on the metagenomic reads sorting process per their origin in the recipient's post-FMT stool metagenome. After the sorting process the resulting reads categories were taxonomically annotated using the MetaPhlAn3 tool. The presence of the donor-derived microbes in the all metagenomes of 
each patient are shown in Figure 5A. Read counts of donor-derived microbial species found in the patient's metagenomes using RECAST software are presented in Supplementary Table S6.

Frequency of occurrence of donor-derived microbes in post-FMT recipient metagenomes were presented in Figure 5B. A total, 67 donor-derived microbial species were identified that successfully colonized the intestines of IBD patients $(9.25 \pm 5.78$ per CD patient; $20.00 \pm 13.68$ per UC patient). The list of top 10 microbes-colonizers included bacteria which distinguished in the previously analysis as healthy-related such as $F$. prausnitzii $(\mathrm{n}$ samples $=20), B$. adolescentis $(\mathrm{n}$ samples $=18), B$. uniformis $(\mathrm{n}$ samples $=17)$, Eubacterium rectale $(\mathrm{n}$ samples $=16), C$. aerofaciens $(\mathrm{n}$ samples $=14)$, P. copri $(\mathrm{n}$ samples $=12), B$. longum (n samples $=12), B$. vulgatus $(\mathrm{n}$ samples $=12)$, A. putredinis $(\mathrm{n}$ samples $=12)$, Bacteroides thetaiotaomicron $(\mathrm{n}$ samples $=11)$. It is worth noting that $F$. prausnitzii, B. adolescentis, C. aerofaciens, and Eubacterium rectale are the most successful microbes in the UC group ( $\mathrm{n}$ samples $=12$ ) while $B$. uniformis in the CD group (n samples $=9$ ).

Other colonization differences in dependence on disease CD vs UC were also observed. A higher occurrence of donorderived microbial colonization regardless of the bacterial species was found (Fisher's exact test, $p<0.001$ ). Obvious significance species-specific colonization patterns in dependence on disease were not found. Species colonizing UC patients can colonize CD patients too, only at a lower frequency. Some bacteria have a decrease in colonization events in CD groups for example $C$. aerofaciens $(\mathrm{CD}=2 ; \mathrm{UC}=12)$ or $A$. hadrus $(\mathrm{CD}=0 ; \mathrm{UC}=6)$ however such tendencies are not statistically significant after applying Benjamini-Hochberg correction for multiple hypothesis testing. Interestingly, those UC patients (UC3, UC8, UC15, UC22) stool samples with more donor-derived bacteria were found closest to the donor and to the healthy population.

In addition, more donor-derived microbes occur per UC metagenomes in comparison with CD samples (see Figure 5C; Wilcoxon rank-sum test, $\mathrm{p}<0.05$ ). The tendency to increase donor-derived reads in the UC group in comparison with the $\mathrm{CD}$ group also were observed (see Figure 5D; Wilcoxon rank-sum test, $\mathrm{p}=0.1$ ).

According to analysis of variance using PERMANOVA the microbes-colonizers content are significantly associated with donor and disease variables $\left(\mathrm{R}^{2}=0.42\right.$, adj. $\mathrm{p}<0.05 ; \mathrm{R}^{2}=0.13$, adj. $\mathrm{p}<0.05$ respectively; Aitchison distance, 10000 permutations).

\section{Discussion}

The world scientific community is conducting extensive research of fecal microbiota transplantation (FMT) to study its efficacy in treatment of the patients with a variety of disorders including inflammatory bowel diseases (IBD). However, despite large-scale already implemented studies, the accumulation of additional experimental data and the use of new analysis methods can be useful in identifying biological insights that may be important for a better understanding of the IBD pathogenesis nature as well as the possible therapeutic effect of FMT.

FMT has enhanced efficacy in the treatment of recurrent Clostridioides difficile infection (CDI) which consist of around $90 \%^{5}$ whereas the FMT in IBD cases is less successful and around 20-40\%6,7. The efficacy of FMT therapy for IBD can be enhanced through the development of clinical protocols based on combinations of FMT and inflammation control

medications ${ }^{8-10}$. In this study, we report about the experience of using FMT in conjunction with IBD common anti-inflammatory therapy. According to monitoring of patients, we observed 75\% (6/8) of clinical remissions ratio in the CD group and 83\% in the UC group (5/6) during observation time. A decrease in disease activity was observed for all patients included in this study. There were no significant side effects associated with the procedure that could be distinguished from the IBD symptoms. Our pilot study has demonstrated that FMT in conjunction with inflammatory control could be a good development in IBD modern therapy. However, large-scale studies and extended cohorts are required to provide a clearer understanding of optimal treatment protocols and its safety.

The study of changes in the microbiota composition of IBD patients following FMT procedure is also the object of close study. One of the main criteria characterizing gut microbiota of healthy subjects is alpha-diversity, which describes the richness of the microbial community. IBD patients usually show a decrease in this score compared to the healthy controls and increase after FMT in comparison with pre-FMT state ${ }^{13-16}$. However, according to our results the patients' gut microbiota alpha-diversity has not increased after FMT and fluctuated on the healthy donors-like level during observation time. Moreover, there was no difference between samples of healthy donors and patients before FMT. It is possible that the effect of alpha diversity increasing depends on the initial patients' clinical state, the feces aggregation state or other unidentified factors.

Changing the microbial profile to a healthy-like type may be a marker of decreasing disease activity ${ }^{12,17,18}$. In IBD patients, there is a significant decrease in beneficial bacteria that are usually extensive abundant in healthy controls and an increase in potentially opportunistic species ${ }^{12,17-19}$. Some researchers suggest that the general microbial biomarker of IBD is decreasing in F. prausnitzii and other SCFA-producing microbes ${ }^{20-23}$. F. prausnitzii has strong anti-inflammatory activity and has been shown to reduce colitis symptoms in a variety of studies ${ }^{24-26}$. Thus, we evaluated the movement of the IBD patients microbiota structure to donor samples and the healthy population after FMT procedure. We observed a shift towards donor and healthy-related gut microbial profiles in the UC group but not in the CD group. Moreover, the change in the microbial content 
was associated with an increase in metabolic potential only in the UC group too. It is worth noting that the microbiome of the $\mathrm{CD}$ patients has been changed, however, in a different way from the described above.

Another important point of analysis is to assess the possible contribution of the donor microbiota to changes in the microbial profiles of patients. The movement towards the donor and even more the movement towards the healthy population cannot be considered a clear marker of FMT-related microbial colonization. Thus, the engraftment of donor-derived microbial strains in IBD patient's post-FMT metagenomes were analyzed. The top five microbes which engraft recipients included $F$. prausnitzii, $B$. adolescentis, B. uniformis, Eubacterium rectale, and $C$. aerofaciens. It is worth noting that $F$. prausnitzii, B. adolescentis, $C$. aerofaciens, and $E$. rectale are the most successful in the UC group while $B$. uniformis in the CD group.

Interestingly, the best colonizers are defined as most associated with healthy conditions as well as described in literature as the most beneficial bacteria. For example, in addition to $F$. prausnitzii which was described above, B. adolescentis also have anti-inflammatory effects due to regulating Treg/Th2 response ${ }^{27}$. Besides many other successful microbes-colonizers, three bifidobacteria species can be associated with strong beneficial composition of gut microbial community including anti-inflammatory, anti-cancer and other helpful effects ${ }^{28-31}$. It has been shown that bifidobacteria interact with human immune cells and modulate certain pathways, including innate and adaptive immune processes ${ }^{29}$.

According to the recent our "restructuring hypothesis", the more prevalent and competent microbes in the human population ("successful gut microbes") are forming a post-FMT recipient microbial profile ${ }^{32}$. Finding that these "successful gut microbes" are the most beneficial (that could have happened under the influence of host-microbial co-evolution for increasing both fitness), a possible explanation of the FMT therapeutic effect can be formulated: FMT improves the recipient's gut microbiota composition by selecting the most host-beneficial of the donor-derived and recipient-derived microbial diversity. Moreover, FMT may transmit/restore the lost symbiotic bonds of healthy-related strains which can increase the summary beneficial effect $^{33}$. Improved in this manner gut microbiota can mediate to the broad spectrum of observed positive effects such as weight

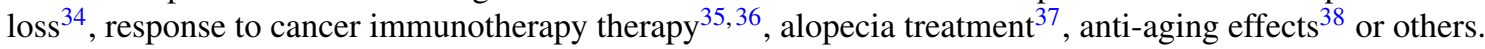

To support this process, "successful gut microbes" must be survive in the presence of host-produced microbial peptides ${ }^{32,39}$. Thus, resistance to intestinal colonization is formed due to the evolutionarily established symbiotic interaction: host forms environmental conditions where "successful gut microbes" gain an advantage over opportunistic microorganisms. Continuing this thought, dysbiosis in CD can be induced by exploiting this "evolution vulnerability". Paneth cells' function may be aggravated, which causes a decrease in the production of host-derived antimicrobial peptides ${ }^{40}$ (or production of altered peptides to which "successful microbes" are sensitive). In other words, environmental conditions change where "successful gut microbes" no longer gain an advantage and hence opportunistic microbes compete better with them. The number of butyrate-producers is reduced, which leads to a decrease in the supply of enterocytes, mucosa degradation and as a result to inflammation. In inflammation, some opportunistic microbes can gain even more benefit from the use of inflammation-related resources and further increase inflammation through positive feedback ${ }^{41}$. Interestingly, the shift in the gut microbial balance in UC may have a different mechanism that could be more likely to be corrected by anti-inflammation medications. Thus, our observed disease-dependent microbial engraftment may reflect the difference in the molecular mechanisms of dysbiotic conditions forming in IBD different types.

\section{Conclusions}

The presented study assessed the probable effect on the gut microbiota changes in CD and UC patients after undergoing FMT together with basic anti-inflammatory therapy. There have been promising clinical results that may be useful for the development of new treatment regimens for IBD. We note differences in the patients microbial profile changes, as well as a decrease in colonization events in the CD group compared to UC. We assumed that the engraftment (by analogy with differences in degradation degree of gut microbiota) can be explained by differences in the pathogenesis of CD and UC. It is also possible that colonization characteristics can be considered as a marker of changes in conditions within the recipient's intestine. In addition, we observed that major butyrate-producers and other beneficial bacteria are the most successful colonizers. Perhaps, the evolutionary formed ecological relationship between the gut microbiota and the host can be related to the therapeutic mechanism of FMT.

\section{Methods}

\section{Study design}

This is a prospectively and retrospectively observational study evaluating patients $(\mathrm{n}=14,10$ males and 4 females, 37.1 \pm 13.7 y.o.) with ileal $(n=7)$ or ileocolonic $(n=1)$ Crohn's disease (CD) and 6 ulcerative colitis (UC) since Jule, 2018 to September, 2019. All eligible subjects provided written informed consents before participation. All patients were examined and treated at the department of gastroenterology of Federal Research and Clinical center of Physical-Chemical Medicine, Federal Medical and Biological Agency of Russia, Moscow, Russian Federation in accordance with relevant 
guidelines and regulations and Helsinki declaration. Disease symptom severity was assessed using the Harvey-Bradshaw Index (HBI) for CD patients ${ }^{42}$ and Simple Clinical Colitis Activity Index (SCCAI) for UC patients ${ }^{43}$. Inclusion criteria were: patients who were diagnosed as $\mathrm{CD}$ or UC by a combination of typical clinical symptoms, endoscopic, and histological criteria; patients with active CD (HBI assessment) and UC (SCCAI assessment). Diagnostic criteria of CD and UC cases was made according to actual guidelines [ECCO-ESGAR Guidelines, 2018, 2019 (www. ecco-ibd. eu/ publications/ecco-guidelines-science/published-ecco-guidelines.html)]. Summary data about the patients included in the study are present in Supplementary Table S1.

Six patients were primarily diagnosed $(5 \mathrm{CD}, 1 \mathrm{UC}$ ). During hospitalization, the patients were prescribed (or accepted/corrected) basic therapy: 5-aminosalicylic acid (5-ASA) monotherapy or combination of 5-ASA and glucocorticoids with doses consistent with the disease severity and according to actual clinical guidelines [ECCO-ESGAR Guidelines, 2018, 2019]. Patients were excluded if aged $<18$ years, accompanied by other severe diseases, including other intestinal diseases, e.g., C. difficile infection, malignant neoplasm, cardiopulmonary failure, and serious liver and kidney disease, and follow-up less than 3 months.

The study design scheme is shown in Figure 1A.

\section{Donor screening}

Healthy persons (aged $22.0 \pm 3.6$ years, 2 males and 4 females) were selected as fecal donors. They received a usual, balanced, European diet over the entire period of the study and were clinically evaluated according to a protocol recommended by the European Consensus Conference on Faecal Microbiota Transplantation in Clinical Practice ${ }^{5}$. The donors were recruited according to the following exclusion criteria: 1) family history of IBD or cancers; 2) infectious diseases; 3) metabolic syndrome; 4) arterial hypertension; 5) obesity; 6) diabetes mellitus; 7) antibacterial therapy in at least 6 month prior the study; 8) tattoo in at least 6 month prior the study.

The selected donors have been in good health, before the fecal collection, being subjected to a routine medical checkup. No dyspepsia or other stool abnormalities were reported within the last year. Routine clinical and laboratory examinations such as clinical blood cell counts, biochemical blood analysis, evaluation of lymphocyte sub-populations, and urine analysis included physical, chemical and microscopy of sediment analysis showed no abnormalities.

Donor blood was examined according to the following: (1) Syphilis; (2) hepatitis B and C; (3) parasites testing including protozoa and helminths. Donor stool has been thoroughly examined: (1) testing for intestinal opportunistic microbial species; (2) testing for antibiotic resistant bacteria such as MRSA, VRE, ESBL- and carbapenemase-producing Klebsiella pneumoniae and Escherichia coli; (3) study of fecal calprotectin; (4) examination for $C$. difficile toxins A and B; (5) PCR testing for enteropathogenic viruses: CMV, Epstein-Barr virus, HSV-1, HSV-2, HHV-6, human adenoviruses (types B, C, E), noroviruses (types 1,2), rotaviruses (types A, B, C), enterovirus; (6) parasites testing including protozoa and helminths. Implemented clinical testing didn't show abnormalities.

\section{Stool sampling and FMT procedure}

For feces collected in an ambulance, the donor received $100 \mathrm{ml}$ of a magnesium sulfate solution and within $1-1.5$ hours the defecation happens. Immediately after that, $150 \mathrm{ml}$ of saline $(0.9 \%)$ was added to the donor sample for every $30 \mathrm{~g}$ of feces and mixed for uniform consistency. In the presence of solid undigested particles in the feces, the solution was filtered using a paper filter. Glycerin is added to the solution to a final concentration of $10 \%$. The final solution was poured into a plastic container and frozen at $-80^{\circ} \mathrm{C}$. All manipulations were performed during no more than 90 minutes from the donor defecation event. The fecal solution was used within no more than 16 weeks from the date of freezing. Before the FMT, the solution was thawed at room temperature for $4-5$ hours, or in a water bath at $+37^{\circ} \mathrm{C}$ one hour before the procedure.

The $100 \mathrm{ml}$ final donor fecal solution was infused into the jejunum using an endoscope. To expand the jejunum, carbon dioxide insufflation was used. Additionally, it was useful for creating an anaerobic environment. The donor fecal solution was drawn into a syringe; the syringe nozzle was placed in the mouth of the endoscope instrumental channel and slowly injected. After the procedure, the endoscope was removed from the patient's jejunum. Excess carbon dioxide was also removed. After the endoscope removing, the patient was placed in Fowler's position. Being in this position, the patient massaged his abdomen, slowly turning from side to side at $30 \mathrm{~min}$. These movements should help distribute the donor fecal solution in the patient's gut.

FMT procedure was performed in conjunction with the basic therapy. Previously published results suggest that the use of FMT may allow IBD patients to achieve steroid-free remission as well as improve patient response to steroids therapy ${ }^{6-10}$.

Collection of patients stool samples was performed in sterile plastic containers, both before FMT and at different time points later on. Time points included 1) before FMT 2) 2 days after FMT 3) 7 days after FMT 4) 14 days after FMT 5) 30 days after FMT. In total, 48 stool samples of patients ( 24 CD and 24 UC) were collected. Eight stool samples were collected from donors.

The stool sampling schemes are shown in Figure 1B and 1C. 


\section{DNA isolation and shotgun sequencing of stool samples}

DNA isolation from stool samples was performed using a reagent set for nucleic acid isolation (MagNa Pure Compact Nucleic Acid Isolation Kit I) (Roche) according to the manufacturer's instruction. Further DNA was eluted in $50 \mu 1$ of the buffer supplied with the kit and stored at $-18{ }^{\circ} \mathrm{C}$. A total of $250 \mathrm{ng}$ of genomic DNA was taken for shotgun sequencing library preparation. After DNA sonication on Covaris S220 System (Covaris, Woburn, MA, USA), the size (400-500 bp) and quality of fragmented samples were assessed on Agilent 2100 Bioanalyzer (Agilent, Santa Clara, CA, USA) according to the manufacturer's manual. NEBNext Ultra II DNA Library Prep Kit (New England Biolabs, Ipswich, MA, USA) was used for pair-ended library preparation, and NEBNext Multiplex Oligos kit for Illumina (96 Index Primers, New England Biolabs, Ipswich, MA, USA) was used for libraries' indexing. The libraries were quantified by Quant-iT DNA Assay Kit, High Sensitivity (Thermo Scientific, Waltham, MA, USA). DNA sequencing $(2 \times 125$ bp $)$ was performed on the HiSeq 2500 platform (Illumina, San Diego, CA, USA) according to the manufacturer's recommendations.

\section{Data analysis and visualization}

Raw metagenomic data were quality assessed using FastQC [https://github.com/s-andrews/FastQC]. Technical sequences and low-quality bases $(\mathrm{Q}<30)$ were trimmed with the Trimmomatic tool ${ }^{44}$. The human sequences from metagenomic samples were removed by BBMAP ${ }^{45}$. Metagenomic taxonomic profiling was performed using MetaPhlAn $3^{46-48}$. For functional analysis, HUMAnN $2^{49}$ and KEGG database (release $\left.2020-03-09\right)^{50}$ were used. Described computational steps were implemented in the Assnake metagenomics pipeline [https://github.com/ASSNAKE].

Aitchison distance ${ }^{51,52}$ and non-metric multidimensional scaling (NMDS) were used for bi-dimensional visualization. Additional visualization was performed using the DEICODE approach $^{53}$ with add external metagenomes from IBD patients and healthy subjects $(\mathrm{n}=220)^{12}$. The taxonomic profiles of external metagenomes were obtained using MetaPhlAn3. The Songbird $^{54}$ and Qurro ${ }^{55}$ approaches implemented in the QIIME2 framework ${ }^{56}$ were used to discover biomarkers significantly discriminating against the experimental groups. To determine differences in relative abundance of KEGG pathways, the gene set analysis (GSA) from piano Bioconductor package ${ }^{57}$ was used with the following parameters: GSA using "median algorithm" with gene set significance threshold: adj. $\mathrm{p}<0.05$. KO rankings values (log2-fold changes) obtained using the Songbird approach were used as GSA analysis input data. For detecting donor-derived microbes in the post-FMT recipient's metagenomes, the RECAST approach was used ${ }^{32}$.

Data visualization was performed using ggplot2 [https: / /ggplot2.tidyverse.org] and vegan ${ }^{58}$ libraries implemented for GNU/R ${ }^{59}$.

\section{Availability of data and materials}

Raw metagenomic reads for 56 fecal samples from 14 IBD patients and 6 healthy donors sequenced by Illumina HiSeq 2500 are deposited in the NCBI Archive (project ID: PRJNA763503; Web-address: https://www.ncbi.nlm.nih.gov/ bioproject /763503). All additional data and intermediate metagenomic analysis results were attached to the article as supplementary materials.

\section{List of abbreviations}

FMT: fecal microbiota transplantation. CD: Crohn's disease. UC: ulcerative colitis. IBD: inflammatory bowel disease. CDI: Clostridioides difficile infection. 5-ASA: 5-aminosalicylic acid. HBI: Harvey-Bradshaw Index. SCCAI: Simple Clinical Colitis Activity Index. MRSA: methicillin-resistant Staphylococcus aureus. VRE: vancomycin-resistant enterococci. ESBL: extended-spectrum beta-lactamase. PCR: polymerase chain reaction. CMV: cytomegalovirus. HSV: herpes simplex virus. HHV: human herpesvirus. KEGG: Kyoto Encyclopedia of Genes and Genomes. NMDS: non-metric multidimensional scaling. GSA: gene set analysis.

\section{Authors contributions}

EIO - general data analysis strategy; experimental data analysis and visualization; interpretation of obtained results; wrote the manuscript. TNK - management of CD and UC clinical cases; contribution to the design of the FMT clinical research protocol; performing FMT clinical procedure; contribution to the interpretation of obtained results. DEF - development and support of the bioinformatics computational protocols; data quality control management; the explorational analysis of obtained metagenomes. KMK - supervising DNA isolation and shotgun sequencing. VAV, DIB - performed DNA isolation and shotgun sequencing. RIM - performing FMT clinical procedure. SVL, DNA, IVM - support of the clinical data interpretation and management; contribution to the manuscript preparation. ESZ - donors and recipients clinical data management. AVP - the design of the FMT clinical research protocol; management of donors and recipients clinical data; supervising the collection 
of stool biobank; supervising FMT treatment procedure. VMG - research idea; development of a general research concept; contribution to supervising the FMT project. ENI - development of a general research concept; supervising the FMT project; supervising the design of the FMT clinical research protocol; contribution to the interpretation of obtained results; contribution to the manuscript preparation.

\section{Fundings}

We thank the Center for Precision Genome Editing and Genetic Technologies for Biomedicine, Federal Research and Clinical Center of Physical-Chemical Medicine of Federal Medical Biological Agency for providing computational resources for this project.

\section{Acknowledgment}

We thank the hospital and laboratory staff for participating in this study.

\section{Ethics declarations}

\section{Ethics approval and consent to participate}

This study was approved by the ethical committee of the Federal Research and Clinical Centre of Physical and Chemical Medicine (Protocol No. 2017/02, Apr 13, 2017). Before the start of the study, all donors and patients signed an informed consent.

\section{Consent for publication}

Not Applicable.

\section{Competing interests}

The authors declare that they have no competing interests.

\section{References}

1. Alatab, S. et al. The global, regional, and national burden of inflammatory bowel disease in 195 countries and territories, 1990-2017: a systematic analysis for the global burden of disease study 2017. The Lancet Gastroenterol. \& Hepatol. 5, 17-30, DOI: https://doi.org/10.1016/S2468-1253(19)30333-4 (2020).

2. Ungaro, R., Mehandru, S., Allen, P. B., Peyrin-Biroulet, L. \& Colombel, J.-F. Ulcerative colitis. The Lancet 389, 1756-1770, DOI: https://doi.org/10.1016/S0140-6736(16)32126-2 (2017).

3. Torres, J., Mehandru, S., Colombel, J.-F. \& Peyrin-Biroulet, L. Crohn's disease. The Lancet 389, 1741-1755, DOI: https://doi.org/10.1016/S0140-6736(16)31711-1 (2017).

4. Marchesi, J. R. et al. The gut microbiota and host health: a new clinical frontier. Gut 65, 330-339 (2016).

5. Cammarota, G. et al. European consensus conference on faecal microbiota transplantation in clinical practice. Gut 66, $569-580$ (2017).

6. Costello, S. P. et al. Effect of fecal microbiota transplantation on 8-week remission in patients with ulcerative colitis: a randomized clinical trial. Jama 321, 156-164 (2019).

7. Sokol, H. et al. Fecal microbiota transplantation to maintain remission in crohn's disease: a pilot randomized controlled study. Microbiome 8, 1-14 (2020).

8. Cui, B. et al. Fecal microbiota transplantation through mid-gut for refractory c rohn's disease: Safety, feasibility, and efficacy trial results. J. gastroenterology hepatology 30, 51-58 (2015).

9. Cui, B. et al. Step-up fecal microbiota transplantation strategy: a pilot study for steroid-dependent ulcerative colitis. $J$. translational medicine 13, 1-12 (2015).

10. Cui, B. et al. Step-up fecal microbiota transplantation (fmt) strategy. Gut microbes 7, 323-328 (2016).

11. Mocanu, V. et al. Repeated fecal microbial transplantations and antibiotic pre-treatment are linked to improved clinical response and remission in inflammatory bowel disease: A systematic review and pooled proportion meta-analysis. $J$. clinical medicine 10, 959 (2021).

12. Franzosa, E. A. et al. Gut microbiome structure and metabolic activity in inflammatory bowel disease. Nat. microbiology 4, 293-305 (2019). 
13. Mosca, A., Leclerc, M. \& Hugot, J. P. Gut microbiota diversity and human diseases: should we reintroduce key predators in our ecosystem? Front. microbiology 7, 455 (2016).

14. Vaughn, B. P. et al. Increased intestinal microbial diversity following fecal microbiota transplant for active crohn's disease. Inflamm. bowel diseases 22, 2182-2190 (2016).

15. Paramsothy, S. et al. Specific bacteria and metabolites associated with response to fecal microbiota transplantation in patients with ulcerative colitis. Gastroenterology 156, 1440-1454 (2019).

16. Clooney, A. G. et al. Ranking microbiome variance in inflammatory bowel disease: a large longitudinal intercontinental study. Gut 70, 499-510 (2021).

17. Huttenhower, C., Kostic, A. D. \& Xavier, R. J. Inflammatory bowel disease as a model for translating the microbiome. Immunity 40, 843-854 (2014).

18. Halfvarson, J. et al. Dynamics of the human gut microbiome in inflammatory bowel disease. Nat. microbiology 2, 1-7 (2017).

19. Pascal, V. et al. A microbial signature for crohn's disease. Gut 66, 813-822 (2017).

20. Sokol, H. et al. Faecalibacterium prausnitzii is an anti-inflammatory commensal bacterium identified by gut microbiota analysis of crohn disease patients. Proc. Natl. Acad. Sci. 105, 16731-16736 (2008).

21. Sokol, H. et al. Low counts of faecalibacterium prausnitzii in colitis microbiota. Inflamm. bowel diseases 15, 1183-1189 (2009).

22. Machiels, K. et al. A decrease of the butyrate-producing species roseburia hominis and faecalibacterium prausnitzii defines dysbiosis in patients with ulcerative colitis. Gut 63, 1275-1283 (2014).

23. Lopez-Siles, M., Duncan, S. H., Garcia-Gil, L. J. \& Martinez-Medina, M. Faecalibacterium prausnitzii: from microbiology to diagnostics and prognostics. The ISME journal 11, 841-852 (2017).

24. Miquel, S. et al. Identification of metabolic signatures linked to anti-inflammatory effects of faecalibacterium prausnitzii. MBio 6, e00300-15 (2015).

25. Quévrain, E. et al. Identification of an anti-inflammatory protein from faecalibacterium prausnitzii, a commensal bacterium deficient in crohn's disease. Gut 65, 415-425 (2016).

26. Lenoir, M. et al. Butyrate mediates anti-inflammatory effects of faecalibacterium prausnitzii in intestinal epithelial cells through dact3. Gut microbes 12, 1826748 (2020).

27. Fan, L. et al. B. adolescentis ameliorates chronic colitis by regulating treg/th2 response and gut microbiota remodeling. Gut microbes 13, 1-17 (2021).

28. Sivan, A. et al. Commensal bifidobacterium promotes antitumor immunity and facilitates anti-pd-11 efficacy. Science 350, 1084-1089 (2015).

29. Ruiz, L., Delgado, S., Ruas-Madiedo, P., Sánchez, B. \& Margolles, A. Bifidobacteria and their molecular communication with the immune system. Front. microbiology 8, 2345 (2017).

30. Skelly, A. N., Sato, Y., Kearney, S. \& Honda, K. Mining the microbiota for microbial and metabolite-based immunotherapies. Nat. Rev. Immunol. 19, 305-323 (2019).

31. Parisa, A. et al. Anti-cancer effects of bifidobacterium species in colon cancer cells and a mouse model of carcinogenesis. PloS one 15, e0232930 (2020).

32. Olekhnovich, E. I., Ivanov, A. B., Ulyantsev, V. I. \& Ilina, E. N. Separation of donor and recipient microbial diversity allows determination of taxonomic and functional features of gut microbiota restructuring following fecal transplantation. Msystems 6, e00811-21 (2021).

33. Rios-Covian, D., Gueimonde, M., Duncan, S. H., Flint, H. J. \& de Los Reyes-Gavilan, C. G. Enhanced butyrate formation by cross-feeding between faecalibacterium prausnitzii and bifidobacterium adolescentis. FEMS microbiology letters $\mathbf{3 6 2}$, fnv176 (2015).

34. Lee, P., Yacyshyn, B. R. \& Yacyshyn, M. B. Gut microbiota and obesity: An opportunity to alter obesity through faecal microbiota transplant (fmt). Diabetes, Obes. Metab. 21, 479-490 (2019).

35. Davar, D. et al. Fecal microbiota transplant overcomes resistance to anti-pd-1 therapy in melanoma patients. Science 371, 595-602 (2021). 
36. Baruch, E. N. et al. Fecal microbiota transplant promotes response in immunotherapy-refractory melanoma patients. Science 371, 602-609 (2021).

37. Rebello, D., Wang, E., Yen, E., Lio, P. A. \& Kelly, C. R. Hair growth in two alopecia patients after fecal microbiota transplant. ACG case reports journal 4 (2017).

38. Boehme, M. et al. Microbiota from young mice counteracts selective age-associated behavioral deficits. Nat. Aging 1-11 (2021).

39. Cullen, T. et al. Antimicrobial peptide resistance mediates resilience of prominent gut commensals during inflammation. Science 347, 170-175 (2015).

40. Wehkamp, J. \& Stange, E. F. An update review on the paneth cell as key to ileal crohn's disease. Front. immunology 11, $646(2020)$.

41. Ormsby, M. J. et al. Inflammation associated ethanolamine facilitates infection by crohn's disease-linked adherent-invasive escherichia coli. EBioMedicine 43, 325-332 (2019).

42. Harvey, R. \& Bradshaw, J. A simple index of crohn's-disease activity. The Lancet 315, 514 (1980).

43. Walmsley, R., Ayres, R., Pounder, R. \& Allan, R. A simple clinical colitis activity index. Gut 43, 29-32 (1998).

44. Bolger, A. M., Lohse, M. \& Usadel, B. Trimmomatic: a flexible trimmer for illumina sequence data. Bioinformatics 30, 2114-2120 (2014).

45. Bushnell, B. Bbmap: a fast, accurate, splice-aware aligner. Tech. Rep., Lawrence Berkeley National Lab.(LBNL), Berkeley, CA (United States) (2014).

46. Segata, N. et al. Metagenomic microbial community profiling using unique clade-specific marker genes. Nat. methods 9 , 811-814 (2012).

47. Truong, D. T. et al. Metaphlan2 for enhanced metagenomic taxonomic profiling. Nat. methods 12, 902-903 (2015).

48. Beghini, F. et al. Integrating taxonomic, functional, and strain-level profiling of diverse microbial communities with biobakery 3. Elife 10, e65088 (2021).

49. Franzosa, E. A. et al. Species-level functional profiling of metagenomes and metatranscriptomes. Nat. methods 15, 962-968 (2018).

50. Kanehisa, M., Furumichi, M., Tanabe, M., Sato, Y. \& Morishima, K. Kegg: new perspectives on genomes, pathways, diseases and drugs. Nucleic acids research 45, D353-D361 (2017).

51. Aitchison, J. On criteria for measures of compositional difference. Math. Geol. 24, 365-379, DOI: https://doi.org/10.1007/ BF00891269 (1992).

52. Aitchison, J. The one-hour course in compositional data analysis or compositional data analysis is simple. In PawlowskyGlahn, V. (ed.) Proceedings of IAMG, 3-35 (in V. Pawlowsky-Glahn (ed.), Proceedings of the III Annual Conference of the International Association for Mathematical Geology (vol. I), CIMNE, Barcelona, Spain, 1997). ISBN 84-87867-97-9.

53. Martino, C. et al. A novel sparse compositional technique reveals microbial perturbations. MSystems 4, e00016-19 (2019).

54. Morton, J. T. et al. Establishing microbial composition measurement standards with reference frames. Nat. communications 10, 1-11 (2019).

55. Fedarko, M. W. et al. Visualizing'omic feature rankings and log-ratios using qurro. NAR genomics bioinformatics 2 , lqaa023 (2020).

56. Bolyen, E. et al. Reproducible, interactive, scalable and extensible microbiome data science using qiime 2 . Nat. biotechnology 37, 852-857 (2019).

57. Väremo, L., Nielsen, J. \& Nookaew, I. Enriching the gene set analysis of genome-wide data by incorporating directionality of gene expression and combining statistical hypotheses and methods. Nucleic acids research 41, 4378-4391 (2013).

58. Oksanen, J. et al. Package 'vegan'. Community ecology package, version 2, 1-295 (2013).

59. Team, R. C. et al. R: A language and environment for statistical computing. R core team (2013). 


\section{Figures}

Figure 1. Study design description (Created with BioRender.com). (A) Schematic visualization of a patient's treatment scheme. The different colors denote different diseases. (B) The longitudinal patient's stool sampling scheme. The point of the FMT procedure is marked with magenta color. (C) Personalizing stool sampling scheme for each patient. The different colors denote different diseases. On the left is the patient IDs.

Figure 2. General changes in taxonomic composition of IBD patients stool metagenomes after fecal transplantation. (A) Stacked bar plot showing the relative abundance of the top phyla identified in IBD patients' stool metagenomes. The $50 \%$ confidence ellipses around the centroids for each group are shown. (B) Non-metric multidimensional scaling biplot obtained using taxonomic profiles (species level) of patients' metagenomes and Aitchison distance. Patient samples grouped by disease and donors samples are shown at different points shapes. (C) Alpha-diversity score (Shannon index) of patient metagenomic samples over time. Green area denotes Shannon index means \pm sd across donors metagenomes. (D) Aitchison distance evaluation between stool metagenomes of baseline and other recipient time points. Aitchison distances between paired metagenomes received from the same donor show by dotted green lines.

Figure 3. General changes in taxonomic composition of IBD patients stool metagenomes after fecal transplantation in the context of donors or external IBD patients and healthy controls. (A) Non-metric multidimensional scaling biplot obtained using taxonomic profiles (species level) of patients' metagenomes and external metagenomes from IBD patients and healthy subjects. Filled dots correspond to the IBD FMT patients, empty dots - external IBD patients and healthy subjects metagenomes. Disease shows by different color dots. Aitchison distance used as a dissimilarity metric. The 95\% confidence ellipses around the centroids for each group are shown. (B) Microbial feature ranking in the context of direction in the Axis1 space. The x-axis values correspond to the Figure 3A Axis 1 values. (C) Temporal changes of IBD patients stool metagenomic profiles in the Figure 3A Axis 1 coordinate space. A decrease in the value corresponds to an approach to a healthy population. (D) Combined graph of stool metagenomes taxonomic profiles of patients movement in space coordinates of Figure 3A Axis 1. (E) Temporal changes of Aitchisons distances between recipients and donors stool metagenomes taxonomic profiles. A decrease in the value corresponds to movement close towards the donor profile. Aitchison distances between paired metagenomes received from the same donor show by dotted green lines. (F) Combined graph of stool metagenomes taxonomic profiles of patients movements in the context of the respective donors taxonomic profiles.

Figure 4. Changes in patients' stool metagenomes taxonomic composition over time points. (A,C) The Songbird taxonomy differentials analysis via 'rank plots'. Y-axis shows microbial species, $\mathrm{x}$-axis shows differentials, which describe the log-fold change in features with respect to time points. (B,D) Qurro 'sample plots"describes feature log-ratios in the context of feature rankings (presented in Figure 4A and Figure 4C). (A,B) - for CD group, (C,D) - for UC group.

Figure 5. Detection of donor-derived microbes engraftment in recipients metagenomes. (A) Donor-derived metagenomic reads per microbial species found in the post-FMT recipients metagenomes. (B) Distribution of the microbes-colonizers by the number of detected engraftment cases. (C) Boxplots show differences between IBD patients groups by the number of microbes-colonizers detected per patient's post-FMT metagenomes. (D) Boxplots show differences between IBD patients groups by the donor-derived reads numbers per patient's post-FMT metagenomes. 


\begin{tabular}{|c|c|c|}
\hline Indicator/Disease & Crohn's disease (CD) & Ulcerative colitis (UC) \\
\hline Gender & $7 \mathrm{M} ; 1 \mathrm{~F}$ & $3 \mathrm{M} ; 3 \mathrm{~F}$ \\
\hline Age & $41.9 \pm 12.0$ & $30.8 \pm 14.2$ \\
\hline Manifestation age & $37.9 \pm 11.0$ & $29.2 \pm 14.5$ \\
\hline CD local & 7 L1; 1 L3 & - \\
\hline CD phenotype & $6 \mathrm{~B} 1 ; 2 \mathrm{~B} 2$ & - \\
\hline UC disease extension & - & $2 \mathrm{E} 1 ; 4 \mathrm{E} 3$ \\
\hline Additional complications & $\begin{array}{l}3 \text { arthritis, } 2 \text { gut resection } \\
(1 \text { in small intestine and } \\
1 \text { in rectum) }\end{array}$ & 1 arthritis \\
\hline Medication history in the last 6 months & $\begin{array}{l}\text { 5-ASA }(n=3), \text { Antimicro- } \\
\text { bials }(n=3), \text { Antidepres- } \\
\text { sants }(n=1), \text { Probiotics } \\
(n=1), \text { Glucocorticoids } \\
(n=1)\end{array}$ & $\begin{array}{l}\text { 5-ASA }(\mathrm{n}=4) ; 5-\mathrm{ASA}+ \\
\text { Glucocorticoids }(\mathrm{n}=1)\end{array}$ \\
\hline Basic therapy & $\begin{array}{l}\text { 5-ASA }(\mathrm{n}=4) ; 5-\mathrm{ASA}+ \\
\text { Glucocorticoids }(\mathrm{n}=4)\end{array}$ & 5-ASA $(n=6)$ \\
\hline Clinical remission ratio & $6 / 8(75 \%)$ & $5 / 6(83 \%)$ \\
\hline
\end{tabular}

Table 1. Summary information about IBD patients included in the study. 

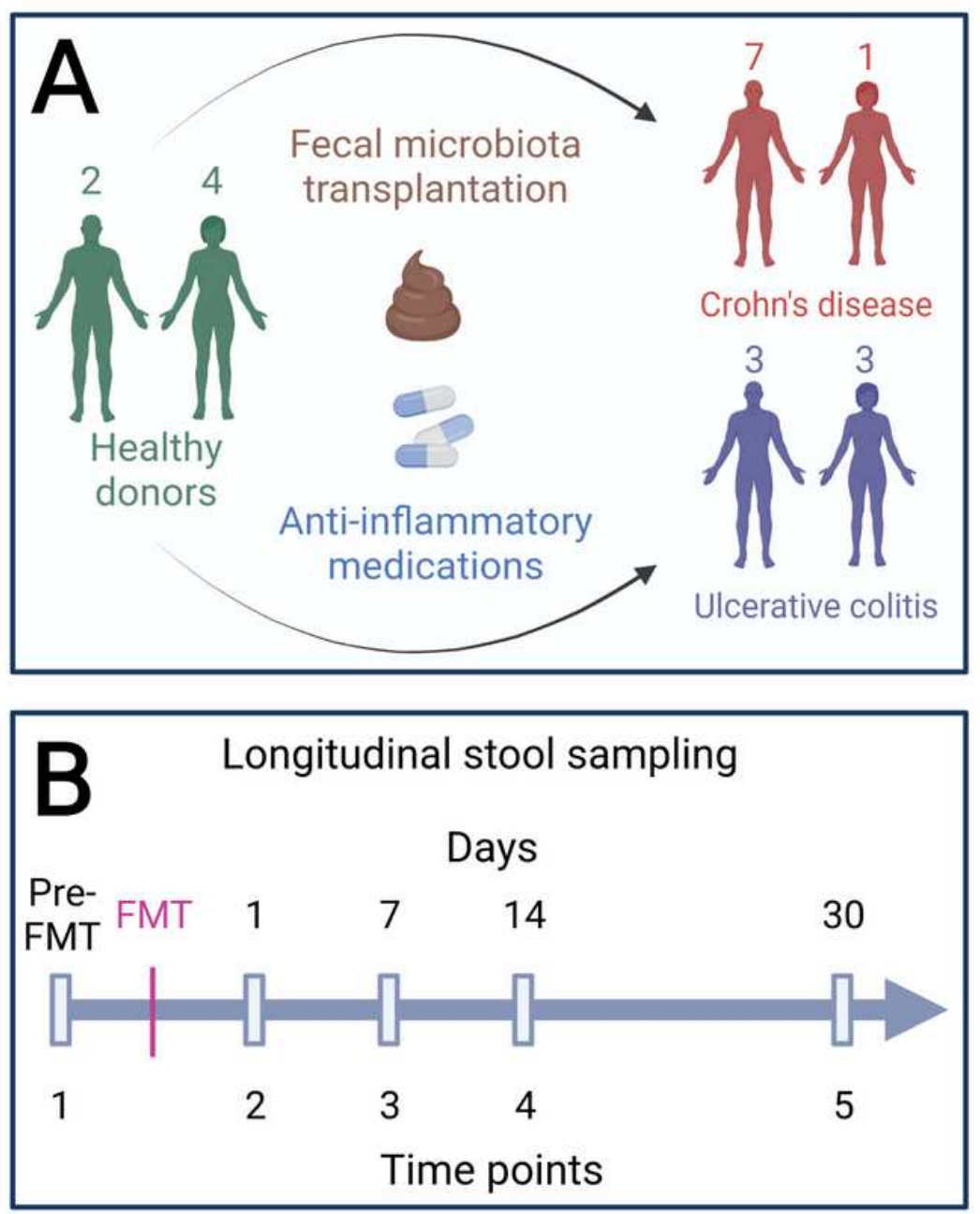

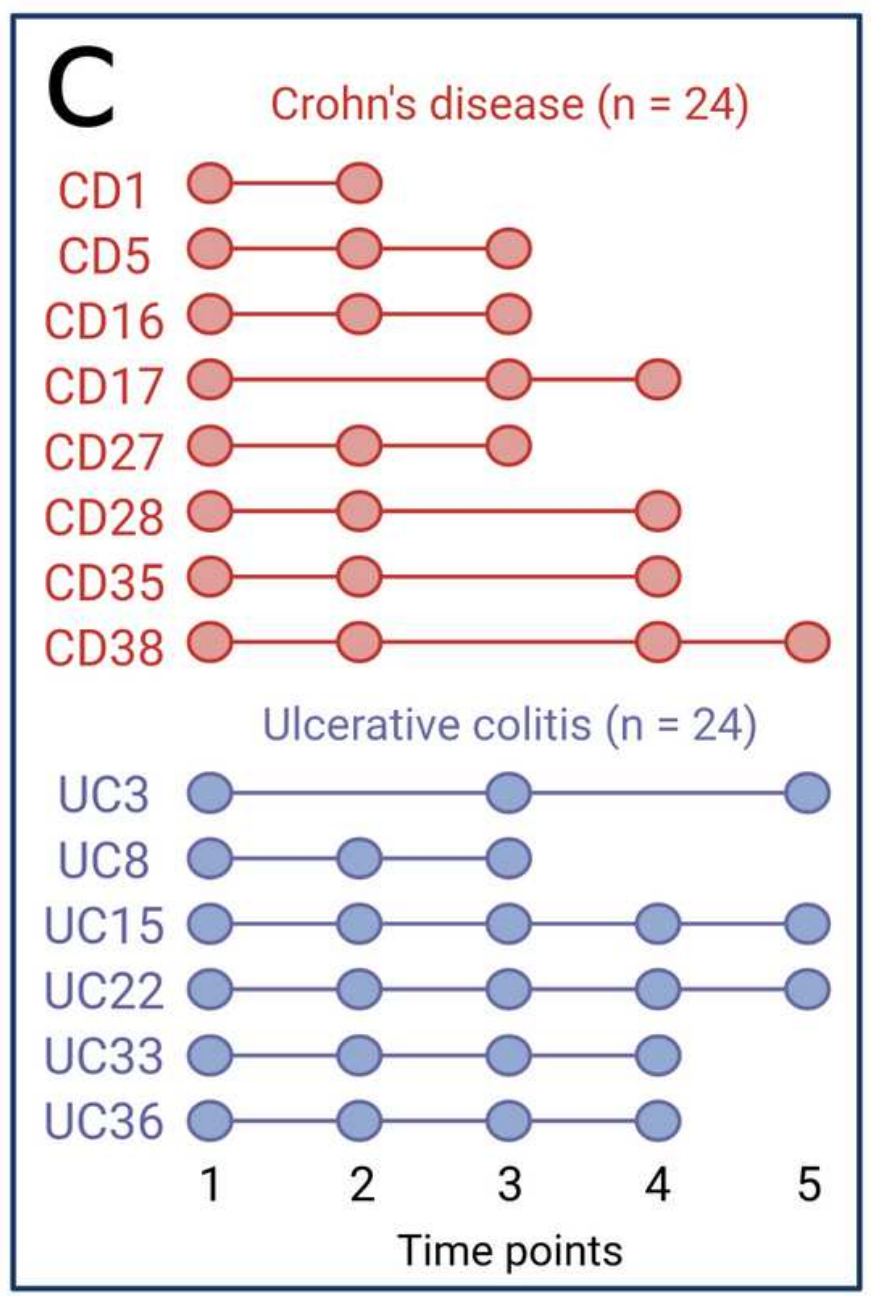

\section{Figure 1}

Study design description (Created with BioRender.com). (A) Schematic visualization of a patient's treatment scheme. The different colors denote different diseases. (B) The longitudinal patient's stool sampling scheme. The point of the FMT procedure is marked with magenta color. (C) Personalizing stool sampling scheme for each patient. The different colors denote different diseases. On the left is the patient IDs. 

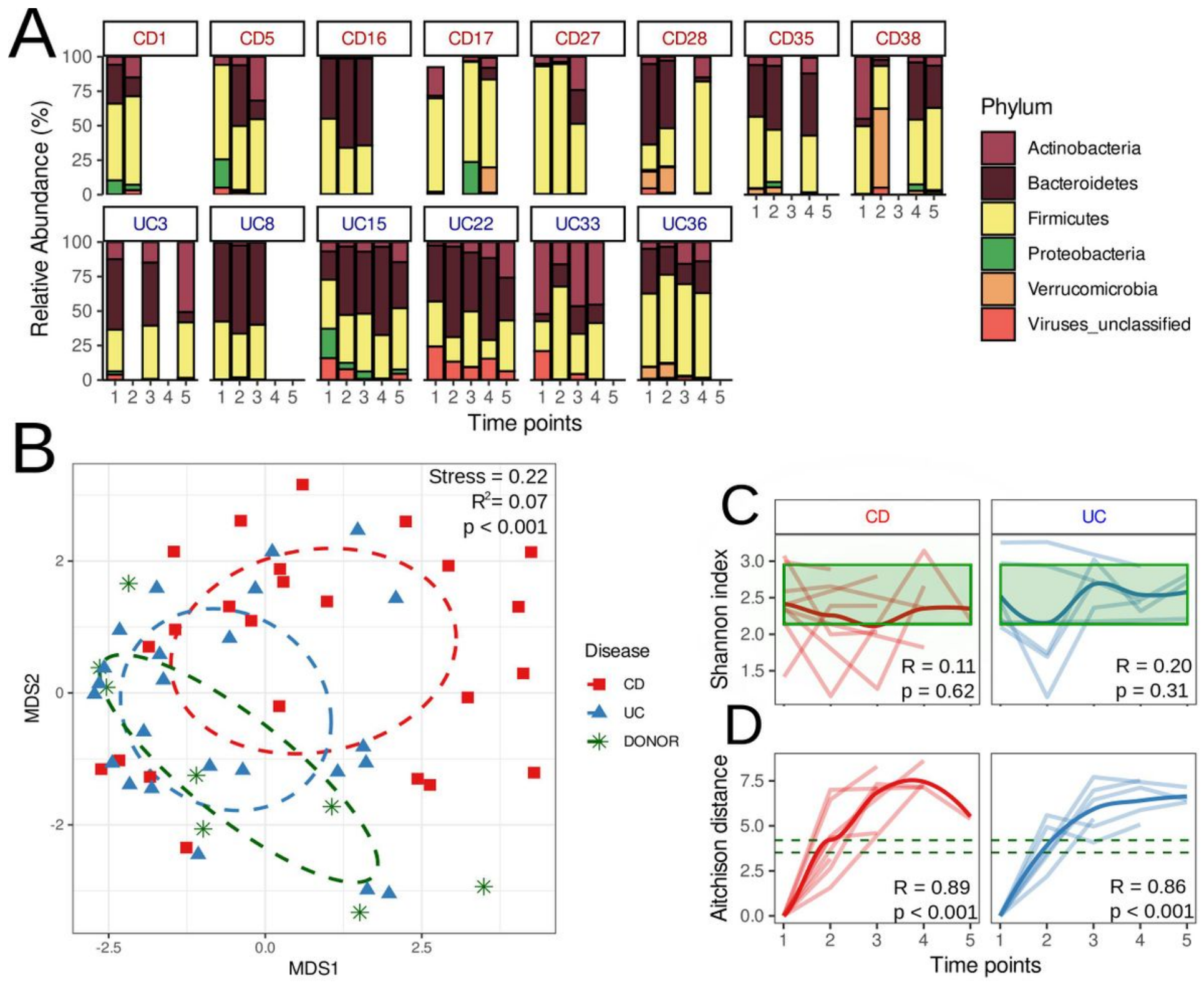

Figure 2

General changes in taxonomic composition of IBD patients stool metagenomes after fecal transplantation. (A) Stacked bar plot showing the relative abundance of the top phyla identified in IBD patients' stool metagenomes. The 50\% confidence ellipses around the centroids for each group are shown. (B) Non-metric multidimensional scaling biplot obtained using taxonomic profiles (species level) of patients' metagenomes and Aitchison distance. Patient samples grouped by disease and donors samples are shown at different points shapes. (C) Alpha-diversity score (Shannon index) of patient metagenomic samples over time. Green area denotes Shannon index means \pm sd across donors metagenomes. (D) Aitchison distance evaluation between stool metagenomes of baseline and other recipient time points. Aitchison distances between paired metagenomes received from the same donor show by dotted green lines. 

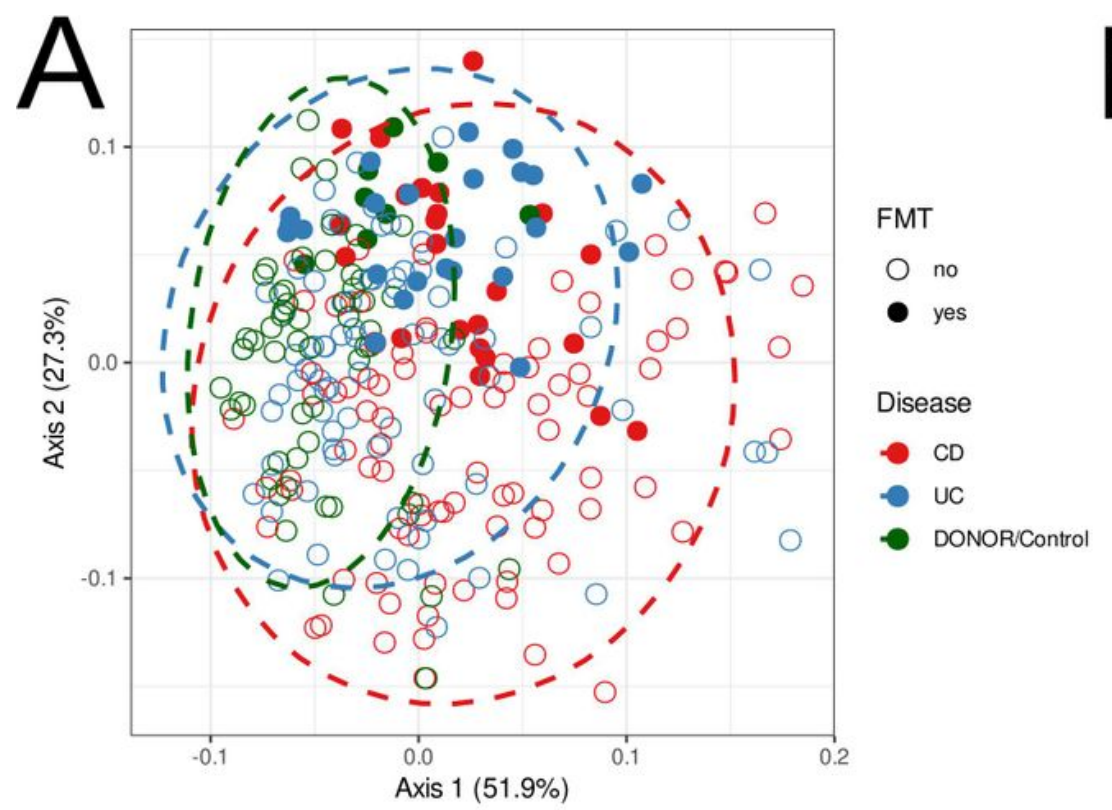

C
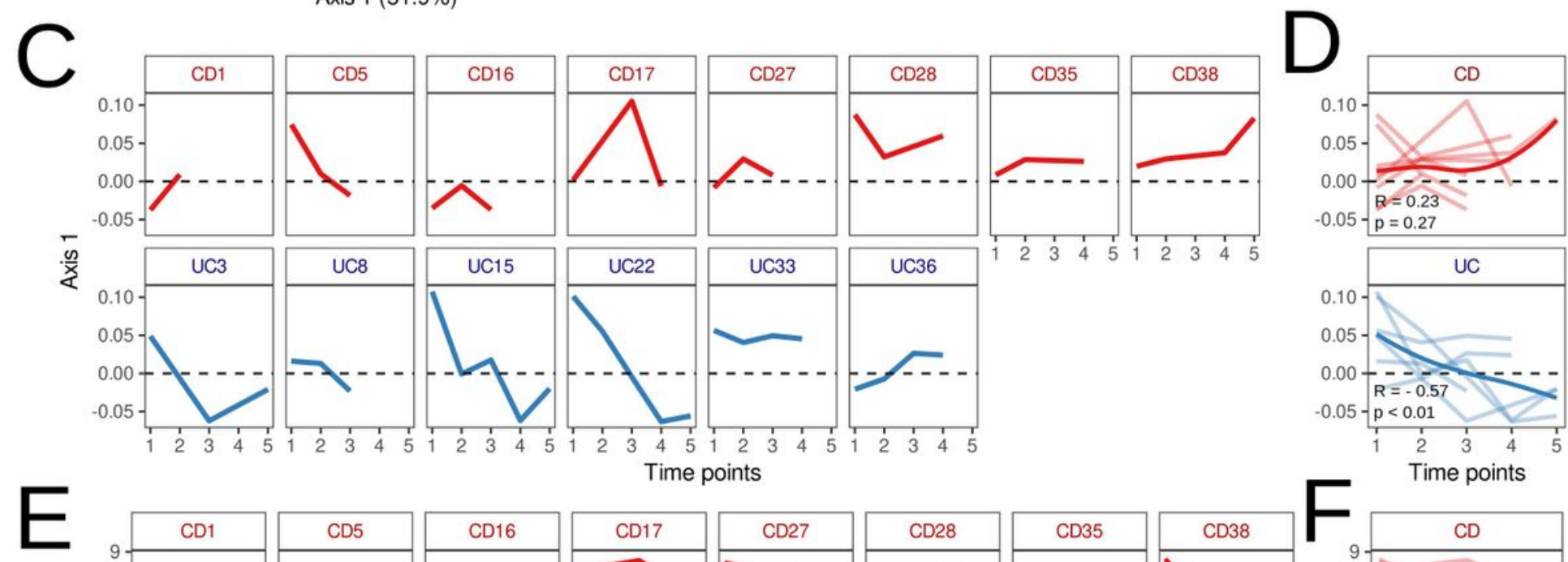

Time points
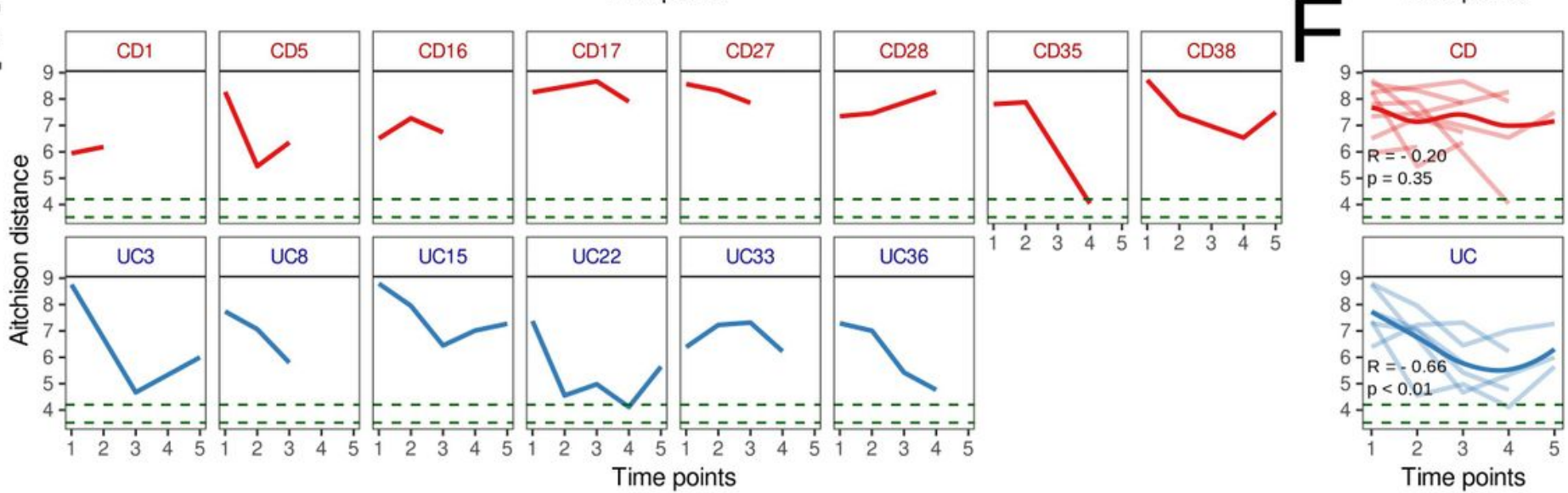

\section{Figure 3}

General changes in taxonomic composition of IBD patients stool metagenomes after fecal transplantation in the context of donors or external IBD patients and healthy controls. (A) Non-metric multidimensional scaling biplot obtained using taxonomic profiles (species level) of patients' metagenomes and external metagenomes from IBD patients and healthy subjects. Filled dots correspond to the IBD FMT patients, empty dots - external IBD patients and healthy subjects metagenomes. Disease shows by different color dots. Aitchison distance used as a dissimilarity metric. The $95 \%$ confidence 
ellipses around the centroids for each group are shown. (B) Microbial feature ranking in the context of direction in the Axis 1 space. The $x$-axis values correspond to the Figure 3A Axis 1 values. (C) Temporal changes of IBD patients stool metagenomic profiles in the Figure 3A Axis 1 coordinate space. A decrease in the value corresponds to an approach to a healthy population. (D) Combined graph of stool metagenomes taxonomic profiles of patients movement in space coordinates of Figure 3A Axis 1. (E) Temporal changes of Aitchisons distances between recipients and donors stool metagenomes taxonomic profiles. A decrease in the value corresponds to movement close towards the donor profile. Aitchison distances between paired metagenomes received from the same donor show by dotted green lines. $(F)$ Combined graph of stool metagenomes taxonomic profiles of patients movements in the context of the respective donors taxonomic profiles.
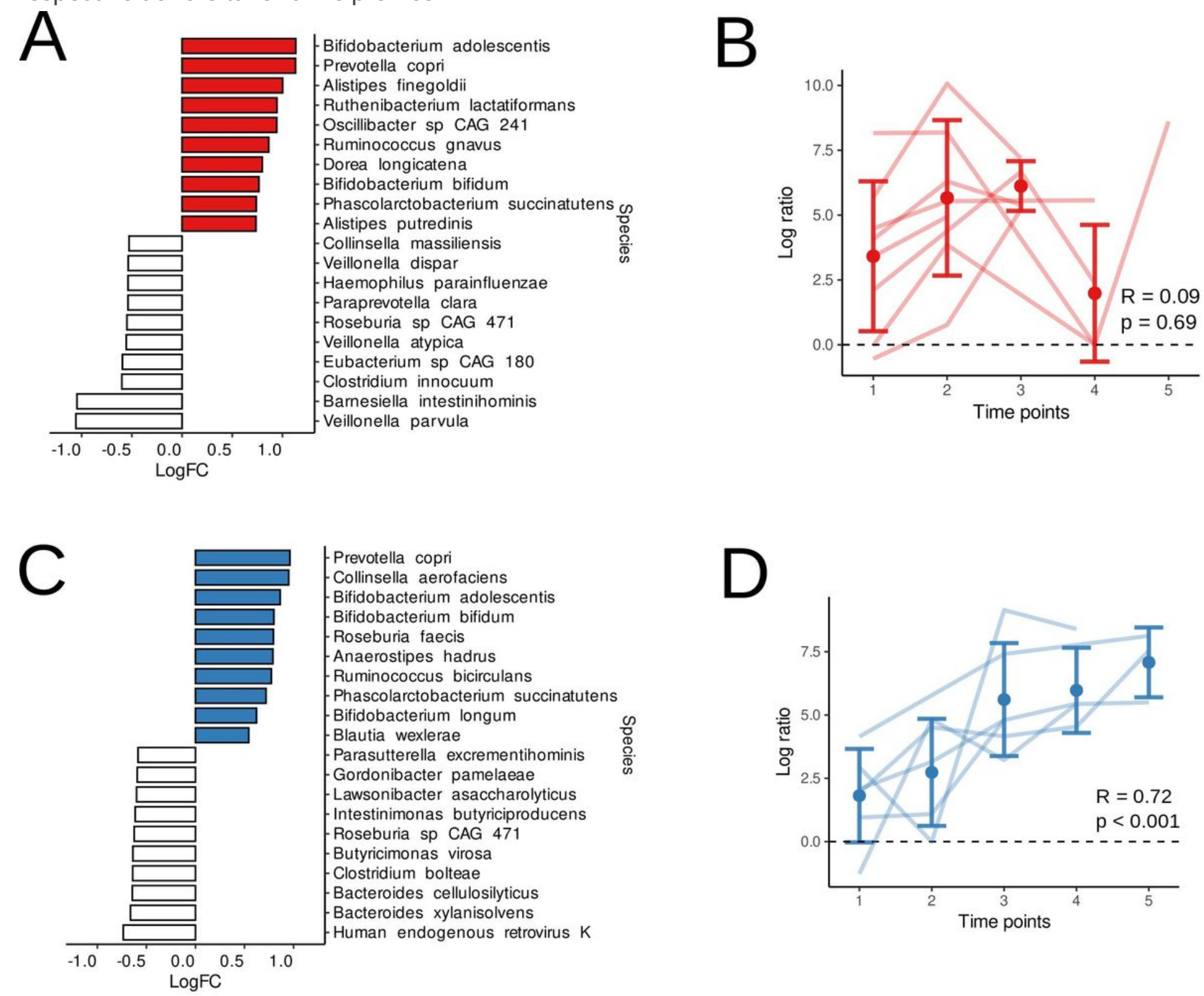

-Prevotella copri
- Collinsella aerofaciens
- Bifidobacterium adolescentis
- Bifidobacterium bifidum
- Roseburia faecis
- Anaerostipes hadrus
- Ruminococcus bicirculans
- Phascolarctobacterium succinatutens
- Bifidobacterium longum
- Blautia wexlerae
- Parasutterella excrementihominis
- Gordonibacter pamelaeae
- Lawsonibacter asaccharolyticus
- Intestinimonas butyriciproducens
- Roseburia sp CAG 471
- Butyricimonas virosa
- Clostridium bolteae
- Bacteroides cellulosilyticus
Bacteroides xylanisolvens
Human endogenous retrovirus $\mathrm{K}$
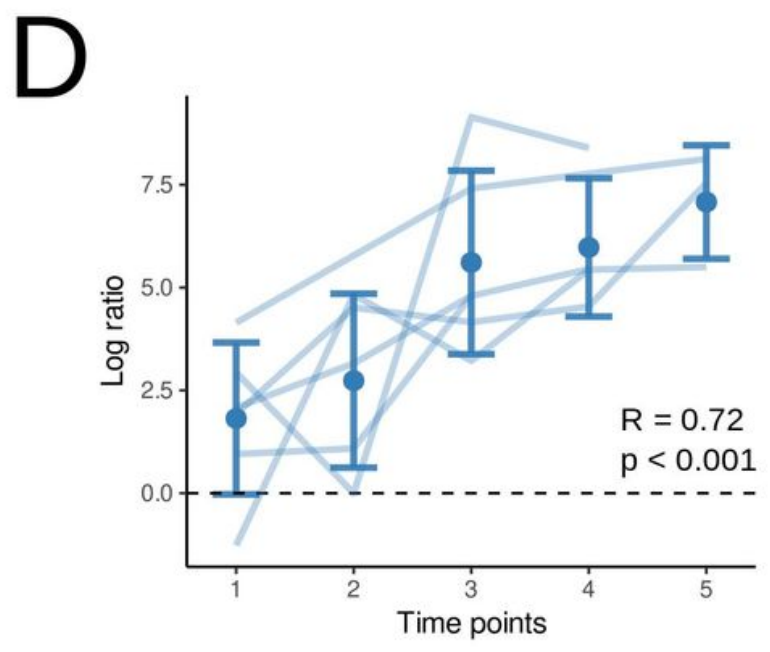

Figure 4 
Changes in patients' stool metagenomes taxonomic composition over time points. $(A, C)$ The Songbird taxonomy differentials analysis via 'rank plots'. Y-axis shows microbial species, $x$-axis shows differentials, which describe the log-fold change in features with respect to time points. (B,D) Qurro 'sample plots"describes feature log-ratios in the context of feature rankings (presented in Figure $4 \mathrm{~A}$ and Figure $4 C)$. (A,B) - for CD group, (C,D) - for UC group.

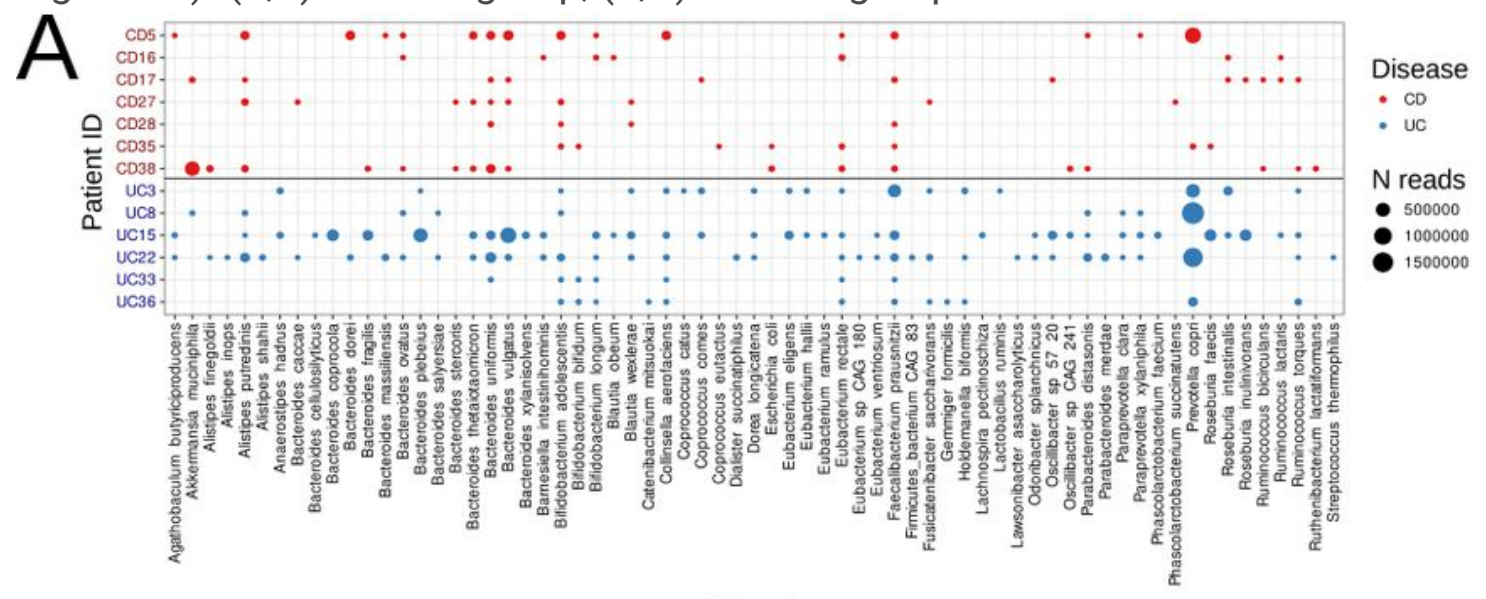

Species
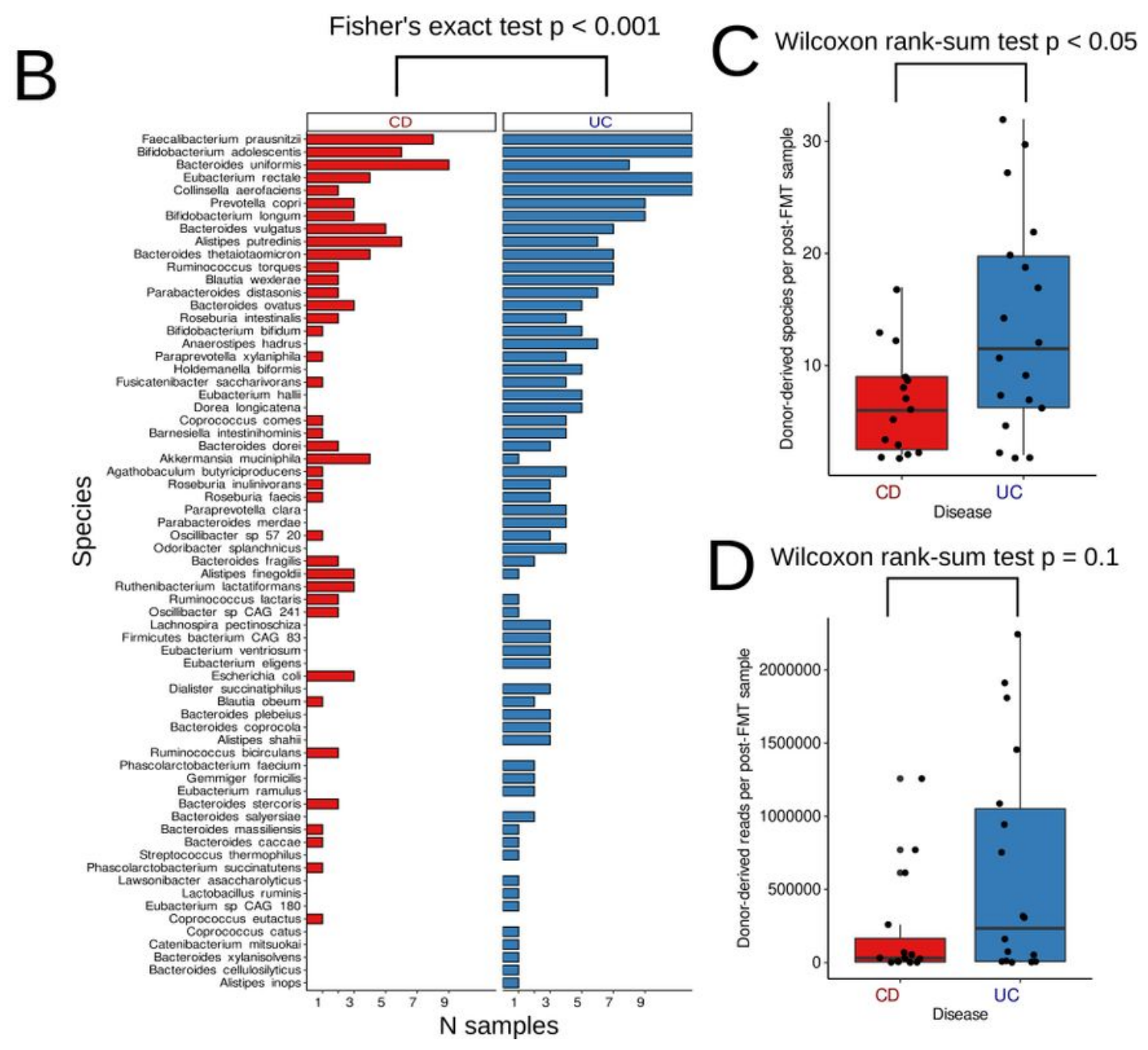

Figure 5 
Detection of donor-derived microbes engraftment in recipients metagenomes. (A) Donor-derived metagenomic reads per microbial species found in the post-FMT recipients metagenomes. (B) Distribution of the microbes-colonizers by the number of detected engraftment cases. (C) Boxplots show differences between IBD patients groups by the number of microbes-colonizers detected per patient's post-FMT metagenomes. (D) Boxplots show differences between IBD patients groups by the donorderived reads numbers per patient's post-FMT metagenomes.

\section{Supplementary Files}

This is a list of supplementary files associated with this preprint. Click to download.

- SupplementaryTables.xlsx 